\title{
Lethality of complex neuronal network in Caenorhabditis elegans nervous system based on cell attacks
}

\author{
Seongkyun Kim, Hyoungkyu Kim, Jaeseung Jeong ${ }^{*}$ \\ From Twenty First Annual Computational Neuroscience Meeting: CNS*2012 \\ Decatur, GA, USA. 21-26 July 2012
}

The aim of this study was to investigate changes in network structural properties and functional perturbations of the C. elegans network which were induced by simulated lesions of the neural network through removals of each single neuron (attacks). We analyzed complete neuronal wiring data (i.e. connectome) of the nematode C. elegans [1] consisting of 279 neurons (nodes) and their connections (edges). We constructed the circular wiring diagram of simply combined network of gap junctions and chemical synapses as shown Figure 1. Then, we measured several measures of complex network properties of directed weighted neuronal network of C. elegans to examine the effect of single node attack: the clustering coefficient, global efficiency, isolated nodes, and reachability [2]. We found that the deletions of motor neurons and interneurons were more effective to the clustering coefficient of the network than the sensory neurons. Eliminations of some interneurons mainly decreased global efficiency, and remarkably increased global efficiencies were induced by each removal of sensory neurons (see Table 1). We suggest that this complex network analysis of the c. elegans connectome is helpful for understanding the potential functions of all neurons, and provide insight into which neurons are crucial for specific functions and which neurons are critical for lethality of the network information processing.

\section{References}

1. Varshney LR, Chen BL, Paniagua E, Hall DH, Chklovskii DB, Sporns O: Structural Properties of the Caenorhabditis elegans Neuronal Network. PLoS Comput Biol 2011, 7(2):e1001066.

2. Watts DJ, Strogatz SH: Collective dynamics of 'small-world' networks. Nature 1988, 393(6684):440-442.

doi:10.1186/1471-2202-13-S1-P110

Cite this article as: Kim et al.: Lethality of complex neuronal network in Caenorhabditis elegans nervous system based on cell attacks. BMC Neuroscience 2012 13(Suppl 1):P110.

\footnotetext{
* Correspondence: jsjeong@kaist.ac.kr

Department of Bio and Brain Engineering, Korea Advanced Institute of Science and Technology (KAIST), Daejeon 305-701, South Korea
}

(c) 2012 Kim et al; licensee BioMed Central Ltd. This is an Open Access article distributed under the terms of the Creative Commons 


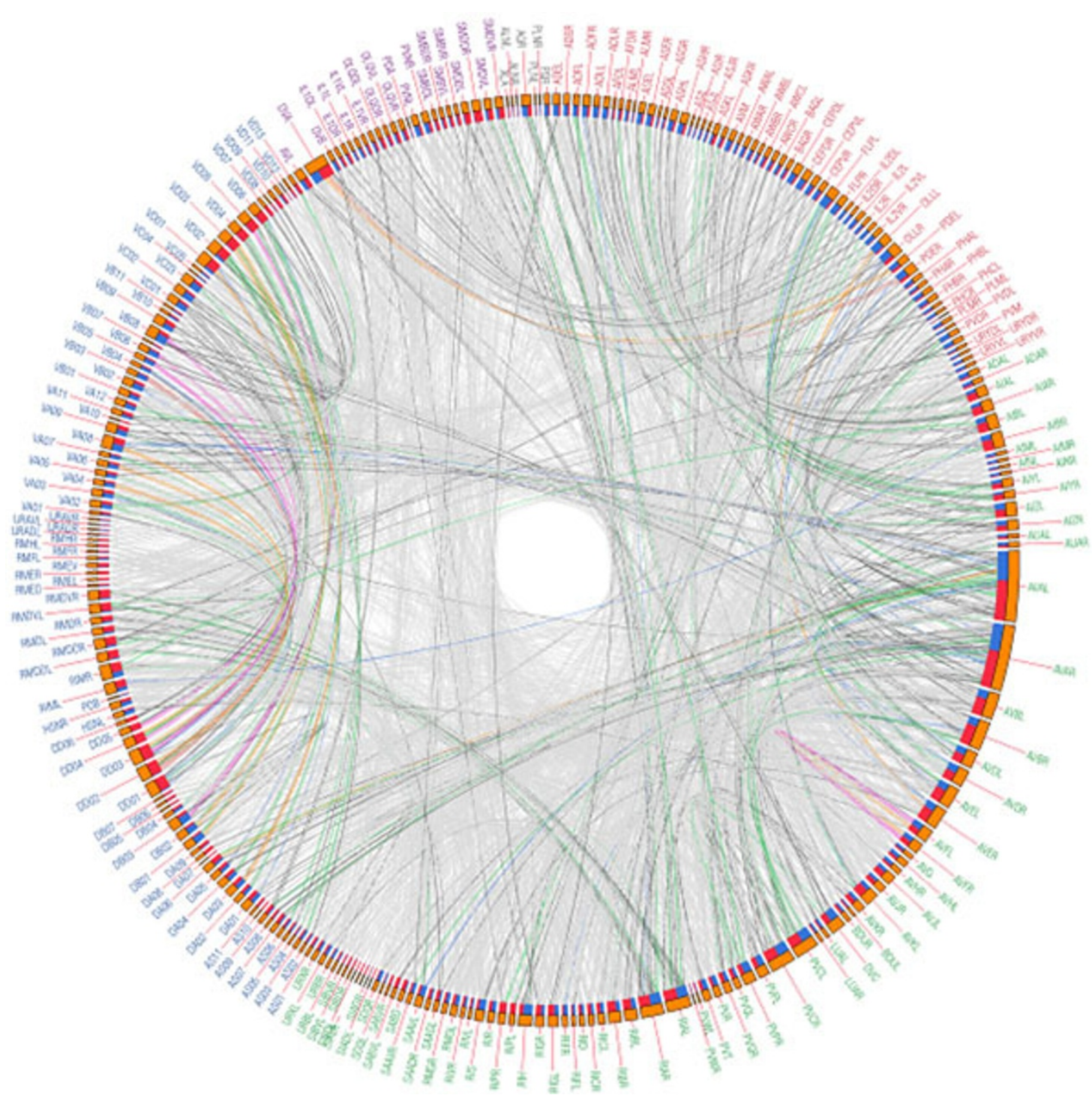

Figure 1 Directed circular wiring diagram of the C. elegans combined network. The colors of the links showed the weights of each connection (Color: Weight ranges; light grey: 1-5, grey: 6-10, green: 11-15, blue: 16-20, orange: 21-25, pink: 26-30, and red: over 30). The color of the name of neuron indicated their neuronal type [1,2] (sensory: red, inter: green, motor: blue, polymodal: purple, and unknown: grey). The links departed from blue segment of a neuron and arrived to red segment of a neuron. The lengths of the orange segments indicated total synaptic strengths (weights) of neurons.

Table 1 The average clustering coefficient and the average global efficiency when a target node deleted. A deletion of one of listed neurons induced remarkable changes in each measure.

\begin{tabular}{|c|c|c|c|c|c|c|c|}
\hline \multicolumn{4}{|c|}{ Clustering Coefficient (CC) } & \multicolumn{4}{|c|}{ Global Efficiency (GE) } \\
\hline \multicolumn{4}{|c|}{ Average of CC in original net: 0.643} & \multicolumn{4}{|c|}{ Average of GE in original net: 1.055} \\
\hline \multicolumn{2}{|c|}{ Increased (176) ${ }^{+}$} & \multicolumn{2}{|c|}{ Decreased (103) } & \multicolumn{2}{|c|}{ Increased (134) } & \multicolumn{2}{|c|}{ Decreased (145) } \\
\hline $\mathrm{DVB}(\mathrm{M})^{++}$ & 0.655 & $\operatorname{AVAR}(I)$ & 0.548 & IL2DR(S) & 1.060 & $\operatorname{AVAL}(I)$ & 0.991 \\
\hline VD10(M) & 0.653 & $\operatorname{AVAL}(I)$ & 0.558 & IL2DL(S) & 1.060 & $\operatorname{AVAR}(I)$ & 1.005 \\
\hline $\mathrm{RID}(\mathrm{M})$ & 0.651 & AS08(M) & 0.624 & $\operatorname{PLNR}(S)$ & 1.059 & DVA $(I)$ & 1.030 \\
\hline HSNR(M) & 0.650 & VA08(M) & 0.631 & URADR(S) & 1.059 & $\operatorname{PVCL}(\mathrm{I})$ & 1.031 \\
\hline $\operatorname{AVL}(\mathrm{M})$ & 0.650 & VB08(M) & 0.631 & URAVR(S) & 1.059 & $\operatorname{PVCR}(I)$ & 1.033 \\
\hline
\end{tabular}

${ }^{+}$The number of the neurons who have increased values of each measurement than the value of original network

${ }^{++}$S: Sensory neuron, I: Interneuron, M: Motor neuron 